\title{
Illusory continuous motion from oscillating positive- negative patterns: implications for motion perception
}

\author{
Stuart M Anstis \\ Department of Psychology, York University, 4700 Keele Street, Downsview, Ontario M3J 1P3, Canada \\ Brian J Rogers \\ Department of Experimental Psychology, University of Oxford, South Parks Road, Oxford OX1 3UD, UK \\ Received 28 July 1981, in revised form 5 November 1986
}

\begin{abstract}
A black and white (positive) grating pattern was superimposed in exact register on its own photographic negative. Four operations were repetitively applied to this positive pattern so that it moved fractionally to the right, grew dimmer, moved back to the left, and grew brighter again. This sequence produced a strong illusion of continuous apparent motion to the right for as long as the cycle was repeated. The small relative motion between the two patterns generated two new illusory effects: enhanced real movement (ERM) and reversed real movement (RRM). The dimming and brightening phases gave rise to reversed apparent movement (RAM). All three effects are attributed to spatial filtering by neural mechanisms, which shifts the effective position of the positive-negative contours.
\end{abstract}

\section{Introduction}

An object which moves smoothly and continuously across the visual field is defined as being in real movement (RM). An image which is flashed in successive positions, as in a movie, can create the illusion of stroboscopic or apparent movement (AM), if the intervals in space and time are appropriate (Anstis 1978, 1980, 1986). In this paper we describe some new illusions of motion which can arise from combinations of real and apparent movement in two patterns when one pattern is the photographic negative of the other.

$\mathrm{RM}$ and $\mathrm{AM}$ are easy to generate with $35 \mathrm{~mm}$ slides projected on to a screen. $\mathrm{RM}$ can be produced by slowly moving the slide carriage of the projector back and forth by hand. AM can be produced by projecting two identical pictures on the screen, overlapping but slightly displaced to be just out of register. A cut between pictures, in which one picture is switched off just as the other is switched on, produces a sharp apparent jump, whereas a dissolve, in which the first picture fades down as the second picture fades up, produces a slow apparent glide.

We have previously reported variations upon reversed apparent movement (RAM) (Anstis 1970; Anstis and Rogers 1975; Rogers and Anstis 1975). Subjects were presented with a white bar which dissolved to a black bar that was shifted a few minutes visual angle to the right. Strong slow apparent movement was reported to the left, ie in a direction toward the earlier stimulus and opposite to the physical displacement. Any pair of black and white pictures could be used, provided that one was the photographic negative of the other, and it made no difference whether the positive or the negative came first. As long as the two pictures were of opposite contrast, the direction of the AM was reversed. Thus a series of dissolves back and forth between the white and black bars gave RAM alternately to the left and to the right. A complete dissolve between the positive and negative pictures was not necessary (see also Gregory and Heard 1982). Any small change in the relative contrasts of the two pictures was sufficient to produce RAM.

Our explanation of RAM was based upon spatial summation or smoothing by neural mechanisms in the visual system (Anstis and Rogers 1975; Rogers and Anstis 1975; Rogers $1976 \mathrm{~b}$ ). We showed that if the profiles of the composite stimulus pattern during 
successive stages of RAM were spatially smoothed, with either a gaussian or a difference of gaussians weighting function, there was a progressive shift of features in the smoothed intensity profile in a direction opposite to that of the actual displacement. These shifts, which were in the same direction as the perceived motion, were present both in the position of the major contours (zero-crossings) and for the peaks and troughs in the smoothed intensity profiles. We concluded therefore that the RAM illusion arose not from particular characteristics of the mechanisms used to extract motion information, but rather from spatial smoothing or filtering by mechanisms at a level before motion itself was extracted. This explanation was supported by the discovery of comparable 'reversed' illusions in both stereoscopic and vernier-alignment tasks which used composite stimulus patterns similar to those used for generating RAM (Anstis and Rogers 1975; Rogers and Anstis 1975). These observations have been confirmed by Gregory and Heard (1982).

In this paper we present two new effects which are related to RAM. We combined these new effects with RAM into a repetitive cyclically moving display, which actually oscillated back and forth through only a few minutes visual angle, but which gave a strong illusion of endless movement to the left (or to the right), as if through many degrees of visual angle. We shall first describe these new effects, and then show how they can be combined into a four-stroke cyclical display.

Normal and reversed AM can be seen during dissolves between two stationary pictures which vary in luminance but do not change their position. The two new effects arise when one picture shifts over another picture, so that the individual pictures vary in position but not in luminance.

\section{Illusions involving real relative motion: movement of a pattern over a copy of itself}

A pattern was made to move with small slow real movement across a stationary copy of itself. The stationary copy could be either an identical positive pattern, or else a photographic negative with all the brightnesses reversed. In pilot work, two projectors shone overlapping slides on to a screen. One slide was kept stationary while the other was moved by manually pushing the slide carriage gently back and forth.

When the moving and stationary patterns were well out of register no unexpected effects occurred. But at the crucial instant when the two patterns came into register, one established and two novel phenomena could be produced.

\subsection{Reduced real movement: pattern moving over a positive copy of itself}

As the moving pattern passed through the in-register position, its speed momentarily appeared to fall to about half (Harris 1980). We attribute this to spatial filtering (Anstis and Rogers 1975; Anstis and Cavanagh 1981), which is thought to blur the two patterns together into a single fused pattern whose composite contours will move at half the speed of the physically moving pattern. When the distance between the patterns exceeded the summatory area of a few minutes visual angle, the moving patterns broke away, and once again two separate patterns were seen. This effect has also been studied by Harris (1980) and by Goldberg and Pomerantz (1981).

\subsection{Enhanced real movement (ERM): pattern moving over a dimmer low-contrast negative copy of itself}

The stationary positive was replaced by a photographic negative, which was dimmed down, and thus reduced in contrast. As the positive pattern moved through the inregister position, its velocity appeared to increase sharply for a brief instant. Then, as the moving pattern moved out of the range of the stationary pattern, it resumed its previous velocity.

This apparent velocity increase (enhanced real movement, or ERM) is caused, in our view, by spatial filtering. When the positive and negative patterns are within a few 
minutes visual angle of each other, their luminance profiles are visually fused into a single profile which effectively moves forward very rapidly, at about twice the speed of the physically moving pattern (figure $1, \mathrm{a} \rightarrow \mathrm{b}$ ).
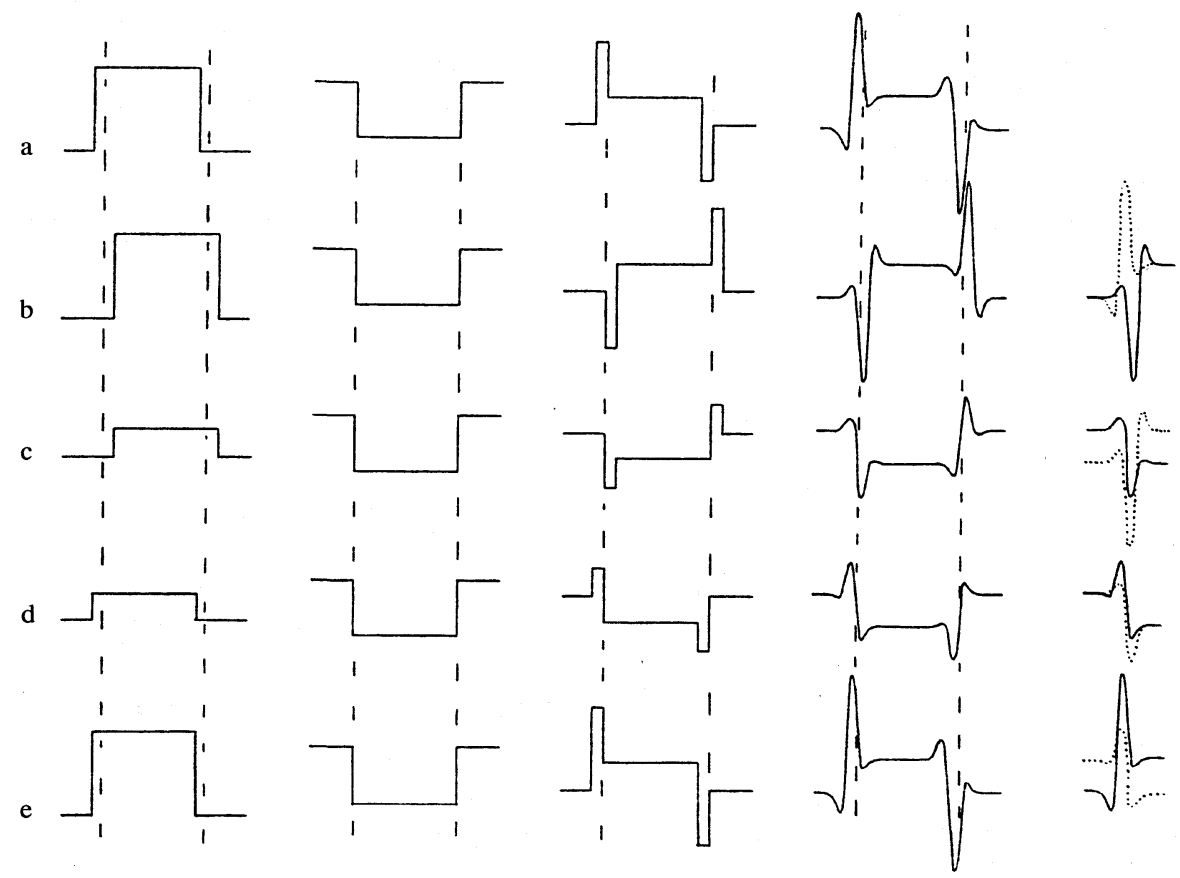

Figure 1. Luminance profiles of the stimuli used in the four-stroke cycle. Rows a-e represent four discrete frames from the continuous sequence (e is the same as a). In the first column, a positive (light) bar starts at a position slightly to the left of the reference position (dashed lines). During the first stage of the cycle it is displaced a small distance to the right $(a \rightarrow b)$. In the second stage its contrast is reduced $(b \rightarrow c)$. In the third stage it is displaced slightly to the left, back to its original position $(\mathrm{c} \rightarrow \mathrm{d})$. In the fourth stage its contrast is increased to its original value $(\mathrm{d} \rightarrow \mathrm{e})$. The second column shows the stationary and unchanging negative (dark) bar in the reference position, upon which the changing positive is superimposed. The third column shows the composite intensity profile at each of the discrete temporal stages $(\mathrm{a}-\mathrm{e})$, formed by adding together the intensity profiles of columns one and two. The fourth column shows the results of convolving the composite intensity profiles of the third column with the difference of gaussians weighting function. The left-hand edges of the smoothed profiles are shown in the fifth column, with the previous profile superimposed (dotted line) in order to show the progressive displacement of the smoothed profile to the right during each stroke.

\subsection{Reversed real movement (RRM): pattern moving over a brighter high-contrast negative copy of itself}

The moving positive was now set to a lower contrast than the stationary negative pattern. As the positive moved through the in-register position, its direction of movement now appeared momentarily to reverse (figure $1, \mathrm{c} \rightarrow \mathrm{d}$ ). If the pattern was moving steadily to the left, it appeared for a moment to reverse its direction and move back to the right (reversed real movement, or RRM), before resuming its motion to the left. In this case, when spatial filtering fuses the two patterns together, their combined luminance profile shows a wave-like ripple running back to the right (figure $1, \mathrm{c} \rightarrow \mathrm{d}$ ).

Thus, we attribute RAM and the two new effects (ERM and RRM) to some kind of spatial filtering or neural blurring in the visual system, generating a neural representation in which the displacement of the contours is reversed or speeded up in comparison with the physical stimulus. 
We shall now show how the two real movement effects, enhanced and reversed, can be combined in a repetitive sequence with the previously reported RAM effects to produce an oscillating stimulus which appears to move steadily and continuously in one direction. We shall say no more about the first effect (reduced real movement), in which a pattern moves over an identical positive copy: in everything that follows a moving or fading positive pattern was superimposed on a stationary negative copy of itself.

\section{A four-stroke cycle: positive pattern oscillating over a stationary negative}

Consider first of all a sequence of four positive patterns (figures $1 \mathrm{a}-1 \mathrm{~d}$, first column; figure $1 \mathrm{e}$ is the same as figure $1 \mathrm{a}$ ), each a white bar on a black ground. The sequence consisted of the following four actions or transitions between pictures, which cycled endlessly. The bar:

$\mathrm{a} \rightarrow \mathrm{b} \quad$ moved through a few minutes visual angle to the right;

$\mathrm{b} \rightarrow \mathrm{c} \quad$ decreased in contrast;

$\mathrm{c} \rightarrow \mathrm{d} \quad$ moved back to the left through a few minutes visual angle to its original position;

$\mathrm{d} \rightarrow \mathrm{e}(\mathrm{a}) \quad$ increased in contrast to its initial value.

When the bar was displayed by itself, it was correctly seen to be oscillating back and forth through a small distance and changing in contrast.

We then superimposed the cycle of four positive patterns on a stationary and unchanging negative pattern or black bar (figure 1, second column). (The position and luminance of the positive pattern always straddled the position and luminance of the negative pattern.) The combination of the two patterns produced the sequence of composite intensity profiles (figure 1 , third column):

$\mathrm{a} \rightarrow \mathrm{b}$ High-contrast white bar moved to the right across a lower-contrast black bar, passing through the in-register position. This is an instance of the ERM described in section 2.2. The movement to the right was perceptually enhanced.

$\mathrm{b} \rightarrow \mathrm{c}$ The white bar, which was now stationary in a position a few minutes visual angle to the right of the black bar, gradually reduced in contrast. This fade or dissolve is an instance of RAM. The perceived motion was in the direction opposite to the black bar's displacement, so apparent motion was seen to the right.

$\mathrm{c} \rightarrow \mathrm{d}$ The low-contrast white bar moved to the left across a negative (black) bar of relatively higher contrast, passing through the in-register position. This is an instance of the RRM described in section 2.3. The direction of the leftward motion was apparently reversed, so apparent motion was seen to the right.

$\mathrm{d} \rightarrow \mathrm{e} \quad$ The white bar, which was now stationary in a position a few minutes visual angle to the left of the black bar, gradually increased in contrast. This fade or dissolve is a further instance of RAM, as was $b \rightarrow c$. It differed from $b \rightarrow c$ in that the white bar was now brightening and to the left of the black bar, instead of dimming and to the right of the black bar. However, the end result was the same, namely apparent movement to the right.

Thus, each of the four strokes in the repetitive cycle gave rise to real or apparent movement to the right. As a result, the stimulus that was actually oscillating back and forth through only a few minutes visual angle appeared to be proceeding steadily and continuously to the right. The continuous motion would be to the left if the sequence were reversed (ie, $d \rightarrow c \rightarrow b \rightarrow a)$.

\section{Method}

The four-stroke cycle was set up on the screen of a Tektronix 604 single-beam oscilloscope equipped with a differential $Z$ (brightness) amplifier. A raster of fine vertical lines was set up on the screen by conventional methods, and a stationary grating of vertical bars was produced by feeding a square (or sinusoidal) waveform to the $Z-$ (negative) input. This produced a 'negative' grating. The 'positive' grating was initially produced by feeding the same input signal to the $Z+$ (positive) input. When the contrasts of the two gratings were adjusted to be equal, this produced essentially a blank screen, since the two gratings cancelled each other out. 
To run the four-stroke cycle, the contrast of the positive grating was driven up and down repetitively by multiplying it with a $1 \mathrm{~Hz}$ triangular waveform, with the use of an electronic analogue multiplier. The position of the positive grating was made to jump back and forth repetitively at $1 \mathrm{~Hz}$ through a few minutes visual angle, by feeding a $1 \mathrm{~Hz}$ square wave into the voltage-controlled phase input of the grating function generator. The $1 \mathrm{~Hz}$ triangular and square-wave signals came from two linked outputs of the same generator, so that they were synchronised to be always $90^{\circ}$ apart in temporal phase angle. Therefore the positive grating executed the desired four actions in repetitive sequence, at a rate of $1 \mathrm{~Hz}$ (figure 1). It:
$\mathrm{a} \rightarrow \mathrm{b} \quad$ jumped a few minutes visual angle to the right (positive-going square wave applied to
$\mathrm{b} \rightarrow \mathrm{c}$
$\mathrm{c} \rightarrow \mathrm{d}$ phase input);
$\mathrm{d} \rightarrow \mathrm{e}$
reduced in contrast (grating waveform multiplied by a negative-going triangular wave); jumped back to the left (negative-going square wave applied to the phase input);

This displacing and contrast-varying positive grating was superimposed on an unchanging negative grating, and it was arranged that the contrast and position of the positive pattern straddled those of the negative pattern. Thus, the contrast of the positive pattern swung alternately higher and lower than that of the negative one, and the position of any given black/white edge in the positive grating moved alternately to the left and right of a corresponding white/black edge in the negative grating. To see what the stimulus looked like, see the demonstration disc in the Appendix.

The outcome of all this was an oscillating display which produced a strong illusion of a continual motion to the right. This illusory motion was measured by a null method of adjustment. The whole display was made to drift physically to the left, as described below, at a rate which the subject could adjust until the display looked stationary. The compensatory motion selected by the subjects was recorded for later analysis.

To keep the pattern stationary on the screen, the timebase of the raster display was triggered by the square wave used to define the luminance profile of the grating (in the conventional way). But in order to make the composite pattern drift across the screen at a rate of $n \mathrm{~Hz}$, with $n$ spatial periods of the grating passing a fixed point every second, the triggering waveform was first electronically shifted in frequency by $n \mathrm{~Hz}$ (Rogers $1976 a)$. This was achieved by feeding a sine wave of the same frequency $F$ as that of the $Z$ modulation square wave into a Surrey Electronics frequency shifter which gave an output of $F+n \mathrm{~Hz}$. The frequency-shifted signal was then used to trigger the timebase of the raster display. A potentiometer under the subject's control allowed the subject to adjust the value of $n$, and hence the physical drift rate of the whole positiveplus-negative pattern.

Two experienced subjects (BJR and MEG) were used. Each subject viewed the display from a distance of $42 \mathrm{~cm}$. The oscilloscope screen subtended a visual angle of $12.3 \mathrm{deg} \times 16 \mathrm{deg}$. Each observer attempted to null the apparent velocity of the fourstroke cycle by means of the method just described, in conditions where the following parameters were varied:

(i) The luminance profiles of the gratings were either sine wave or square wave.

(ii) Viewing was either foveal or peripheral. In the foveal condition the fixation point was in the centre of the screen. In the peripheral condition it lay $9 \mathrm{~cm}(11 \mathrm{deg})$ below the centre of the screen and hence $4 \mathrm{~cm}(5 \mathrm{deg})$ below the bottom edge of the screen.

(iii) Temporal cycling rate was set to $0.5,1$, or $2 \mathrm{~Hz}$.

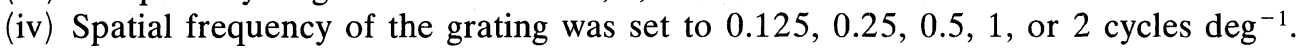
The number of spatial cycles present on the screen was $2,4,8,16$, or 32 , respectively.

(v) Displacement between positive and negative gratings was set to $\pm 2,4$, or 8 min visual angle; in other words, a given black/white edge of the positive grating jumped between 
positions which were located 2,4 , or $8 \mathrm{~min}$ to the left and right of a corresponding white/black edge in the negative grating.

This gave $180(2 \times 2 \times 3 \times 5 \times 3)$ conditions. Two readings were taken in each condition. It was found that the displacement between positive and negative gratings [mentioned under heading ( $v$ ) above] did not make any systematic difference, so the results for all three displacements were averaged together in plotting the results, giving six readings per datum point.

The nulling rates selected by the subject were recorded. Note that the drift rate of a grating can be expressed in two ways: either as a temporal frequency in $\mathrm{Hz}$, namely the number of spatial periods that pass a fixed point in $1 \mathrm{~s}$, or else as a velocity in deg $\mathrm{s}^{-1}$. Velocity is equal to temporal frequency $\div$ spatial frequency, so that if two gratings of 0.25 and 1 cycle $\mathrm{deg}^{-1}$ were both drifting at $1 \mathrm{~Hz}$, their respective velocities would be 4 and 1 deg $\mathrm{s}^{-1}$.

\section{Results}

Results are given in figure 2 in the form of three-dimensional plots in which the apparent drift rate on the ordinate is plotted as a function of spatial frequency of the gratings, $f$, in cycles $\mathrm{deg}^{-1}$ and of temporal frequency or cycling rate of the four-stroke display, $F$, in $\mathrm{Hz}$. The apparent drift rate of the four-stroke cycle was determined by the nulling speed selected by the subject. Note that the units for the ordinate are different in different graphs: in figures $2 a-2 e$ the ordinate is the nulling temporal frequency of the drift in $\mathrm{Hz}$, whereas in figures $2 \mathrm{f}$ and $2 \mathrm{~g}$ it is the nulling velocity of the drift in $\operatorname{deg} \mathrm{s}^{-1}$. All the axes are logarithmic.

Overall, our results can be summarised by saying that the perceived temporal frequency of the drift in $\mathrm{Hz}$ for sinusoidal gratings was constant across spatial frequencies, whereas for square-wave gratings the perceived velocity in deg $\mathrm{s}^{-1}$ was constant across spatial frequencies.

Figures $2 \mathrm{~b}$ and $2 \mathrm{c}$ show that the sinusoidal gratings appeared to drift at a constant rate of one spatial period per temporal cycle, over a wide range of spatial frequencies. The contours on the graph are approximately horizontal. These results indicate a veridical perception of the drifting sinusoids; as the phase diagram of figure 3 shows, each four-stroke cycle physically shifted the grating through one spatial period, so the actual translation rate of the sinusoidal grating in $\mathrm{Hz}$ was close to the stimulus cycling rate.

The square-wave gratings appeared to move more slowly than the sinusoids, especially at low spatial frequencies (figures $2 \mathrm{~d}$ and $2 \mathrm{e}$ ). In particular, the perceived drift rate was quite unrelated to the drift rate of the fundamental. Instead, the square-wave gratings appeared to drift at a constant velocity of about $0.6 \mathrm{deg} \mathrm{s} \mathrm{s}^{-1}$, whatever their spatial frequency, when the stimulus cycled at $1 \mathrm{~Hz}$ (and pro rata when the stimulus

Figure 2 (see facing page). Results from the experiment, averaged for the two observers and given as three-dimensional surfaces in which the apparent drift rate on the ordinate is plotted as a function of spatial frequency of the gratings, $f$, in cycles $\operatorname{deg}^{-1}$ and of temporal frequency of the four-stroke display, $F$, in Hz. All axes are logarithmic. Note that the ordinate in (a)-(e) is the temporal frequency of the nulling drift rate expressed in $\mathrm{Hz}$; in (f) and $(\mathrm{g})$, which are replots of (d) and (e), it is the nulling velocity expressed in deg $\mathrm{s}^{-1}$. (a) Baseline physical motion of a sinusoidal grating undergoing the four-stroke cycle. On each temporal cycle in $\mathrm{Hz}$ the grating physically advances by one spatial period to the right. Results for a sinusoidal grating viewed foveally (b) and peripherally (c). Results approximate to the horizontal contours of the ideal surface shown in (a) (except for some progressive slowing down at lower spatial frequencies). Thus the nulling drift rate in $\mathrm{Hz}$ was relatively independent of spatial frequency. Results for a square-wave grating viewed foveally (d) and peripherally (e). Perceived velocities were slower than for sinusoids. The surface is far from horizontal. However, when the ordinate is changed to nulling velocity in deg $\mathrm{s}^{-1}$ in (f) and (g), surface contours become more horizontal, showing that nulling drift rate in deg $\mathrm{s}^{-1}$ is independent of spatial frequency. 


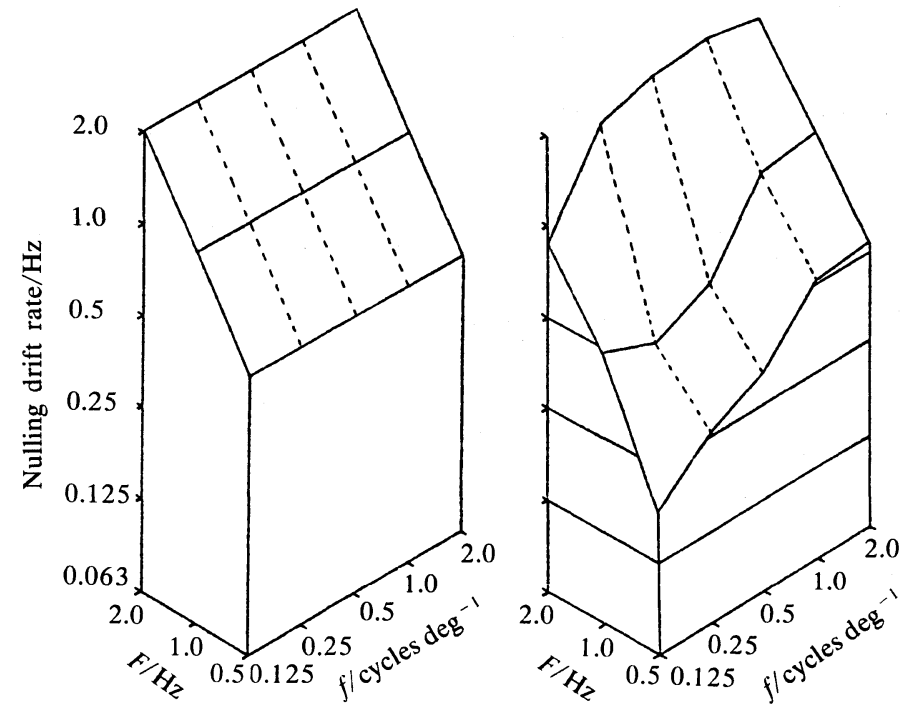

(a)

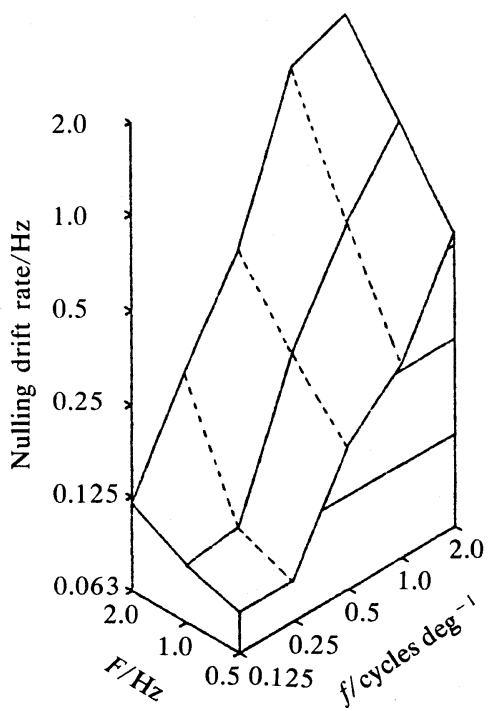

(d)

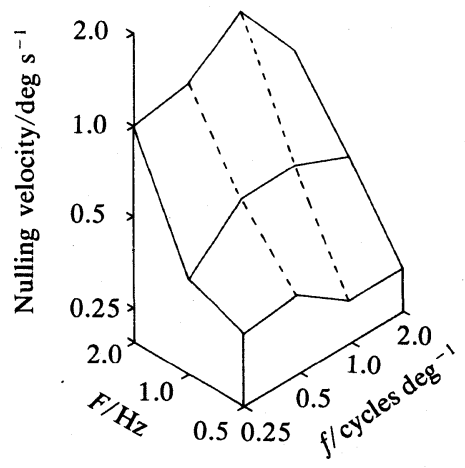

(f)

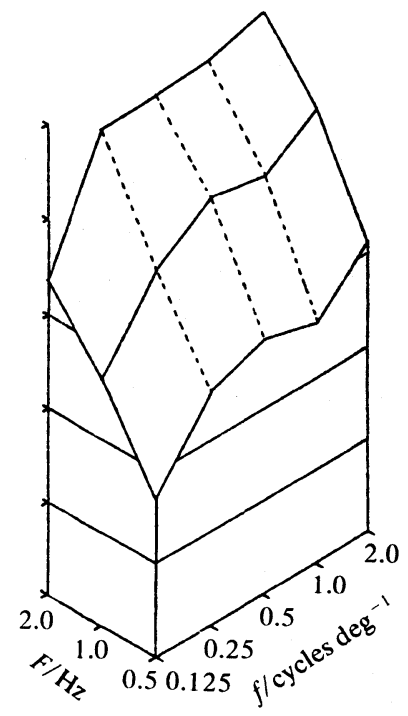

(c)

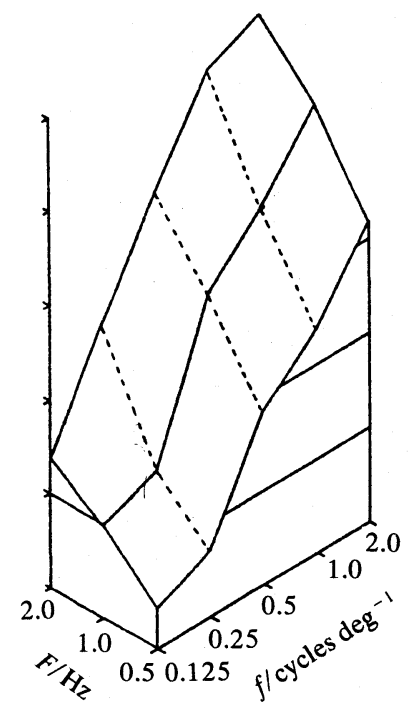

(e)

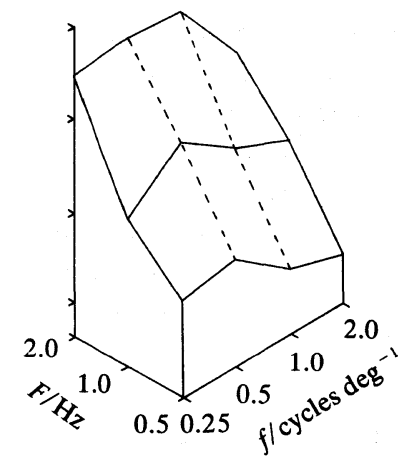

(g) 
cycled at 0.5 or $2 \mathrm{~Hz}$ ). This is best seen in figures $2 \mathrm{f}$ and $2 \mathrm{~g}$, in which the ordinate is now nulling velocity in deg $\mathrm{s}^{-1}$ (instead of temporal drift rate in $\mathrm{Hz}$ ). This transform of the data makes the surface contours on the graph in figures $2 \mathrm{f}$ and $2 \mathrm{~g}$ approximately horizontal, showing that the perceived velocity was independent of spatial frequency.

These results suggest that the perceived velocity of a given edge in a square-wave grating depended only upon events occurring at that edge and was independent of spatial frequency, ie of how many other edges were present and how far away these other edges were. Hence the perceived velocity of the square-wave grating undergoing the four-stroke cycle can be understood better in terms of moving spatial features than in terms of spatial frequency.

\subsection{Four-stroke cycle for a single edge}

A square-wave grating was set up on the screen at such a low spatial frequency that less than a single cycle was visible on the screen. This turned the screen into a bipartite field which was bisected by a vertical contour consisting of a positive black/white edge superimposed on a negative white/black edge. Running the four-stroke cycle gave good apparent motion during each stage of the cycle. Paradoxically, the edge appeared to keep moving to the right without ever getting anywhere.

In summary, our results show that the apparent motion of a sinusoidal grating undergoing the transformations of the four-stroke cycle appeared to move through one spatial period during each four-stroke cycle, and so did a square-wave grating if its spatial frequency was greater than about 1 cycle $\mathrm{deg}^{-1}$ (figures $2 \mathrm{~d}$ and 2e). Square-wave gratings of lower spatial frequencies appeared to move much more slowly at a speed of around $0.6 \mathrm{deg} \mathrm{s} \mathrm{s}^{-1}$, which was independent of the spatial frequency. Also, the spatial displacement between the positive and negative gratings did not make much difference.

\section{Discussion and conclusions}

\subsection{Description in the space domain}

All these apparent motion phenomena can be explained by the changes in the luminance profiles of the stimuli over time. The four-stroke cycle contains two strokes of RAM, one stroke of ERM, and one stroke of RRM. Rogers and Anstis (1975) attributed RAM to spatial smoothing, which effectively shifts the contours during a dissolve in a direction opposite to the physical displacement. We now apply this explanation to enhanced and reversed real movement. Column 3 in figure 1 shows the composite intensity profiles generated by adding a positive (light) bar which changes its relative position and contrast over time (column 1) to its negative (dark) counterpart (column 2). The drawings in column 4 show the same composite intensity profiles as in column 3, but in this case convolved with a difference of gaussians weighting function. In the two strokes of RAM $(b \rightarrow c$ and $d \rightarrow e)$, we can see that the temporally later profile ( $c$ and e) is displaced to the right, whether we compare the positions of the peaks or of the zero-crossings in the smoothed intensity profiles.

The same is true for the displacement of the smoothed intensity profiles in the other two strokes of the cycle $(a \rightarrow b$ and $c \rightarrow d$ ). In both cases, the temporally later profile (b or d) is displaced to the right with respect to the earlier one. This is not surprising in the case of the ERM stroke $(a \rightarrow b)$, since the high-contrast positive pattern is actually displaced to the right during the transition from a to b. However, for the RRM stroke, the displacement of the peaks and the major contours (zero-crossings) of the same polarity in the smoothed intensity profile is still to the right, even though the actual displacement of the white bar is to the left.

Hence all three effects, which make up the four-stroke cycle reported in this paper, can be accounted for by the single assumption that the physical intensity profiles reaching the retina are spatially filtered or smoothed. This shifts the effective positions of the 
stimulus contours so that the illusions will be seen by whatever mechanisms are subsequently used to extract motion.

\subsection{Description in the frequency domain}

Fourier theory tells us that there are two equivalent descriptions of any waveform: its luminance as a function of spatial position, or the amplitudes and phases of its Fourier components. Hence, the four-stroke cycle reported here can be expressed in Fourier terms. Since the spatial-frequency description of the stimulus is equivalent to the spatial description, it may seem superfluous because it makes the same predictions. However, if motion were analysed in separate channels tuned to different spatial frequencies, a Fourier decomposition would allow us to predict the activity in these channels, whereas a spatial description would not. To anticipate, we shall compare the response of such a set of independent channels when confronted with sinusoidal and with square-wave gratings executing the four-stroke cycle.

Consider first a simple sine-wave grating as the (positive) waveform for the fourstroke cycle. In a diagram resembling a Nyquist plot (figure 3A), this sinusoidal grating is represented by a radius vector in a circle. The amplitude or contrast of the grating is given by the length of the vector, and its position or spatial phase by the angular position of the vector. Two superimposed gratings of the same spatial frequency can be depicted by a vector summation, which gives a third vector representing a grating of intermediate contrast and spatial phase (figure 3B).

When a positive sine-wave grating undergoes a four-stroke cycle in the absence of the negative grating, the changes are as shown in figure $3 \mathrm{C}$. The positive grating changes successively as follows: it moves to the right $(a \rightarrow b)$, decreases in contrast $(b \rightarrow c)$, moves back to the left $(c \rightarrow d)$, and increases in contrast $(d \rightarrow a)$. In the polar plot, the locus of the tip of the moving and changing vector traces out a quadrilateral inside the circle. The two dissolves of reversed apparent motion $(b \rightarrow c$ and $d \rightarrow a)$ form the straight radial sides of the quadrilateral, and the two real motion strokes form the arcs of circles, with ERM $(a \rightarrow b)$ at the top side of the quadrilateral and the RRM $(\mathrm{c} \rightarrow \mathrm{d})$ at the bottom.

The usefulness of this plot can be seen when we add the stationary negative unchanging grating. This is $180^{\circ}$ out of phase with the positive grating and appears in the polar plot as a fixed vector pointing vertically down towards ' 6 o'clock'. This displaces the whole quadrilateral downwards, so that it now circumscribes the centre of the circle (figure 3D). Now when the four-stroke cycle is run, the motion of the fundamental is a steady and continuous drift to the right.

This result for a simple sine-wave grating is not surprising since the operation of inverting a sine-wave grating and shifting it to the left by $n^{\circ}$ is the same operation as shifting it to the right by $(180-n)^{\circ}$. The reversed apparent motion for a sine-wave grating is consequently present in the stimulus and would presumably be seen by any visual system.

The situation for a square-wave grating pattern, however, is more complicated. Displacement of the square wave by a fixed spatial extent will displace each Fourier

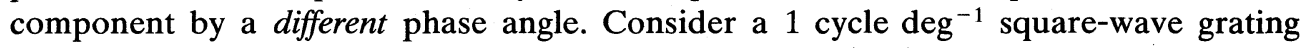
which is inverted in phase and shifted to the left through (say) one tenth of the period of the fundamental. The phase inversion will shift each harmonic through $180^{\circ}$, or 0.5 spatial cycle, but the shift corresponding to one tenth of the period of the fundamental will displace the fundamental and higher harmonics through phase angles of $0.1,0.3$, $0.5,0.7$, etc cycles to the left. Subtracting these from the 0.5 cycle of the phase inversion, we find that the first and third harmonics will be displaced by 0.4 and 0.2 cycle, 
respectively, to the right; the fifth harmonic will be displaced by 0 cycle (ie it will remain stationary); and the seventh harmonic will be displaced by 0.2 cycle to the left. Higher harmonics will move "every which way" (figure $3 \mathrm{E}$ ).

In general, any spatial shift of the square wave will produce a confusing set of shifting harmonics. So the result of a phase inversion plus a small spatial shift will not be RAM of fixed amplitude (as predicted by the earlier account in the space domain) but rather a motley array of apparent movements both to the left and to the right, with a variety of amplitudes. Many of these incoherently moving harmonics can be filtered out by spatial smoothing, leaving only the fundamental and lower harmonics which generally move in the direction of the perceived motion. Thus, spatial filtering plus separate motion mechanisms in different spatial-frequency channels can correctly predict the direction of the perceived RAM.

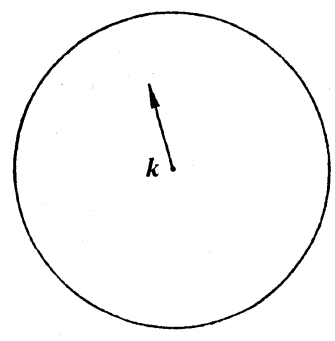

(A)

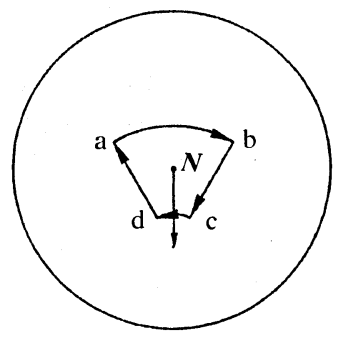

(D)

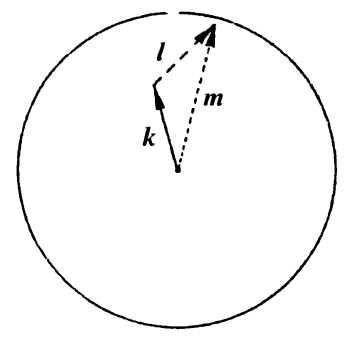

(B)

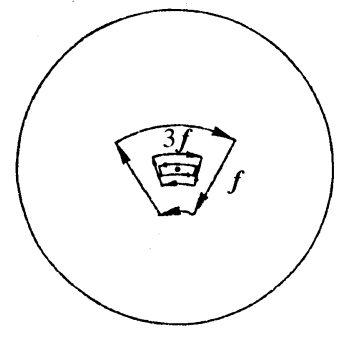

(E)

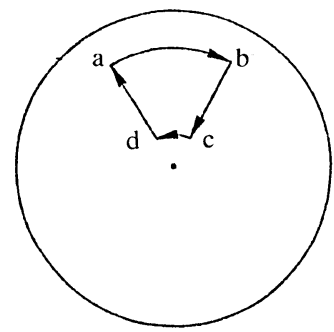

(C)

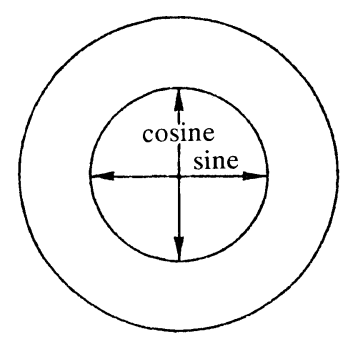

$(\mathrm{F})$

Figure 3. In these vector diagrams, length of vector represents contrast of a grating and angle of vector represents spatial phase or position. (A) shows a single sinusoidal grating $\boldsymbol{k}$. In (B) a second grating $\boldsymbol{l}$ of the same spatial frequency but with a different amplitude and phase is added. The resultant grating $\boldsymbol{m}$ can be predicted by vector addition. During the four-stroke cycle $(C)$, a sine-wave grating: jumps to the right $a \rightarrow b$, decreases in contrast $b \rightarrow c$, jumps back to the left $\mathrm{c} \rightarrow \mathrm{d}$, and increases in contrast $\mathrm{d} \rightarrow \mathrm{a}$. The grating vector describes a quadrilateral in the upper part of the circle. In (D), a static unchanging negative $(\boldsymbol{N})$ is added. The result is that, in vector space, the negative is the downward-pointing vertical vector, and the quadrilateral is offset downwards, so that it circumscribes the centre. The vector rotates continuously clockwise, which in spatial terms is equivalent to a continuous drift to the right. A four-stroke cycle with a square-wave grating which displaces one tenth of a period on each real movement stroke is shown in (E). The vector representing the fundamental, $f$, traces out the same path as the sine-wave vector shown in (D), whereas the higher harmonics trace out quadrilaterals with smaller changes of contrast (length of the straight sides) and with different amounts of displacement in the real movement stages (length of the arcs).

The illusion of continuous motion of a sine wave described by Shadlen and Carney (1986) can be represented as a vector diagram $(F)$ in which the quadrature components (sine and cosine waveforms) are orthogonal vectors. Each component is counterphased in temporal quadrature so that when one component is maximal the other is zero, and vice versa. The locus of the vector sum of two components modulated in this way describes a circle around the centre. In spatial terms, this is equivalent to continuous motion in a single direction. 
However, it does not correctly predict the speed. If the motion of each of the harmonics in the square-wave pattern was handled separately by the visual system, then each harmonic would appear to drift through its own spatial period per temporal cycle (figure $3 \mathrm{E}$ ). Since these spatial periods would be only $\frac{1}{3}, \frac{1}{5}$, $\frac{1}{7}$, etc of the fundamental period, their relative velocities would be $\frac{1}{3}, \frac{1}{5}, \frac{1}{7}$, and so on. Assuming some sort of averaging of velocities in the visual system, these higher harmonics would make the overall motion of the square wave slower than that of a sinusoidal grating, as we found. But the perceived velocity of a square-wave grating would still vary inversely with its spatial frequency (for a constant displacement), which would depress the whole surface of figure $2 \mathrm{~d}$ or $2 \mathrm{e}$ downwards, without altering its slope. This was not what we found. Figure 2d (foveal square-waves) is not simply a downwardly depressed copy of figure $2 b$ (foveal sinusoids), but instead has a very different slope.

These results for the moving square-wave pattern suggest that motion is extracted from the displacements of stimulus edges in the spatial domain rather than from the phase shifts of harmonic components in the frequency domain. This moving-edge model correctly predicts both direction and speed of movement.

Our four-stroke cycle can be compared with an intriguing illusion of continuous motion recently reported by Shadlen and Carney (1986). Their stimulus consisted of two luminance profiles with spatial quadrature relationships between each of the harmonic components. These two profiles were counterphase modulated in time with a $90^{\circ}$ temporal phase difference. Therefore corresponding harmonics in the two profiles were in quadrature both in space and in time. In the case of a simple sinusoidal grating pattern, the spatial quadrature components (sine and cosine waveforms) can be represented on our polar diagram as orthogonal vectors. The result of counterphasing the two patterns in temporal quadrature is a vector which rotates in a circle about the centre like a Lissajous figure (figure $3 \mathrm{~F}$ ). The polar representation of their stimuli resembles our own, although the individual components of their cycle are very different from ours. For instance, where we used a positive and a negative square-wave grating, their two luminance profiles would be one square wave (composed of peaks-subtract sine harmonics) and one approximately triangular waveform (composed of peaks-add cosine harmonics).

Shadlen and Carney reported the impression of continuous motion in one direction from a variety of different luminance profiles such as amplitude- and frequencymodulated gratings, one-dimensional noise texture, and a single line. They showed that a model of motion which uses quadrature spatial and temporal filters can correctly predict the uniform direction of motion actually observed. Nevertheless, they thought that the speed of motion is probably derived from a different mechanism which detects the displacement of features (such as edges), since their quadrature model would incorrectly predict that the harmonics should be seen as moving at many different speeds. Their argument closely parallels our reasons for preferring a spatial (displacement of features) model to explain our results from the four-stroke cycle. For further discussion of motion-sensing models see Adelson and Bergen (1985), and for some recent psychophysical evidence bearing on those models see Moulden and Begg (1986).

Thus Shadlen and Carney conclude that a quadrature model based on spatialfrequency-tuned channels will render direction correctly, but not speed, because the harmonic components in their stimuli move in the same direction but at different speeds. They also conclude that a feature-displacement model will render speed correctly, but not direction, because (they suggest) different features of their stimuli move in different directions, yielding "a Brownian-like motion, or no motion at all". However, we believe that most features in their stimuli, and in ours, do move in the same direction, so that a feature-displacement model will correctly render both speed and direction. We have compared (by superimposition) the spatial quadrature versions 
of the amplitude-modulated grating or the one-dimensional noise texture presented in their figure 2, and found that the overwhelming majority of the peaks and troughs and the major contours of the right-hand profiles are to the left of their nearest neighbours in the left-hand profiles. Hence, there is an overall displacement of corresponding features in their luminance profiles which is consistent with both the perceived direction of motion and the perceived speed. This does not rule out motion detection by Shadlen and Carney's elegant quadrature mechanism, but it opens the door by showing that almost any model which successfully detects real motion will sense motion in their stimuli, and in ours, since there are features of the luminance profiles which really do displace continuously in the perceived direction.

In conclusion, the two new real movement effects reported in this paper, together with the previously reported reversed apparent motion effect, have been combined into a repetitive four-stroke cycle to give the impression of continuous motion in a single direction. All three effects would be expected if there are spatial filters in the visual system which modify the effective positions of the stimulus contours. These contours, successively shifted over time, provide an adequate stimulus to the motion-sensing system.

Acknowledgments. This research was supported by Grant A 0260 to SMA from the Natural Science and Engineering Research Council of Canada (NSERC), and by Grant GR/D 48933 to BJR from the Science and Engineering Research Council of the United Kingdom. We thank Maureen Graham for acting as a subject.

\section{References}

Adelson E H, Bergen J R, 1985 "Spatiotemporal energy models for the perception of motion" Journal of the Optical Society of America 2 284-299

Anstis S M, 1970 "Phi movement as a substraction process" Vision Research $101411-1430$

Anstis S M, 1978 "Apparent movement" in Handbook of Sensory Physiology volume VII Perception eds R Held, H Leibowitz, H-L Teuber (New York: Springer) pp 656-673

Anstis S M, 1980 "The perception of apparent movement" Philosophical Transactions of the Royal Society of London, Series B 290153 -168. Reprinted 1980 in The Psychology of Vision (London: The Royal Society) pp 153-168

Anstis S M, 1986 "Motion in the frontal plane: Sensory aspects" in Handbook of Perception eds K Boff, L Kaufman (New York: John Wiley) chapter 16

Anstis S M, Cavanagh P, 1981 "What goes up need not come down: positive motion aftereffects from moving flickering edges" in Attention \& Performance volume IX eds J Long, A Baddeley (Cambridge: Cambridge University Press) pp 63-77

Anstis S M, Rogers B J, 1975 "Illusory changes of visual depth and movement during changes of contrast" Vision Research 15 957-961

Goldberg D M, Pomerantz J R, 1981 "Models of illusory pausing and sticking" Journal of Experimental Psychology: Human Perception \& Performance 8 547-561

Gregory R L, Heard P, 1982 "Visual dissociations of movement, position and stereo depth: some phenomenal phenomena" Quarterly Journal of Experimental Psychology 35 217-237

Harris C S (ed), 1980 Perceptual Adaptation and Perceptual Coding (Hillsdale, NJ: Lawrence Erlbaum Associates)

Moulden B, Begg H, 1986 "Some tests of the Marr-Ullman model of movement detection" Perception $15139-155$

Rogers B J, 1976a "A technique for generating moving visual stimuli on a CRO" Vision Research $16415-418$

Rogers B J, 1976b Perceptual Consequencies of Temporal and Spatial Summation in the Human Visual System PhD thesis, Bristol University, Bristol, UK

Rogers B J, Anstis S M, 1975 "Reversed depth from positive and negative stereograms" Perception 4 193-201

Shadlen M, Carney T, 1986 "Mechanisms of human motion perception revealed by a new cyclopean illusion" Science 232 95-97 


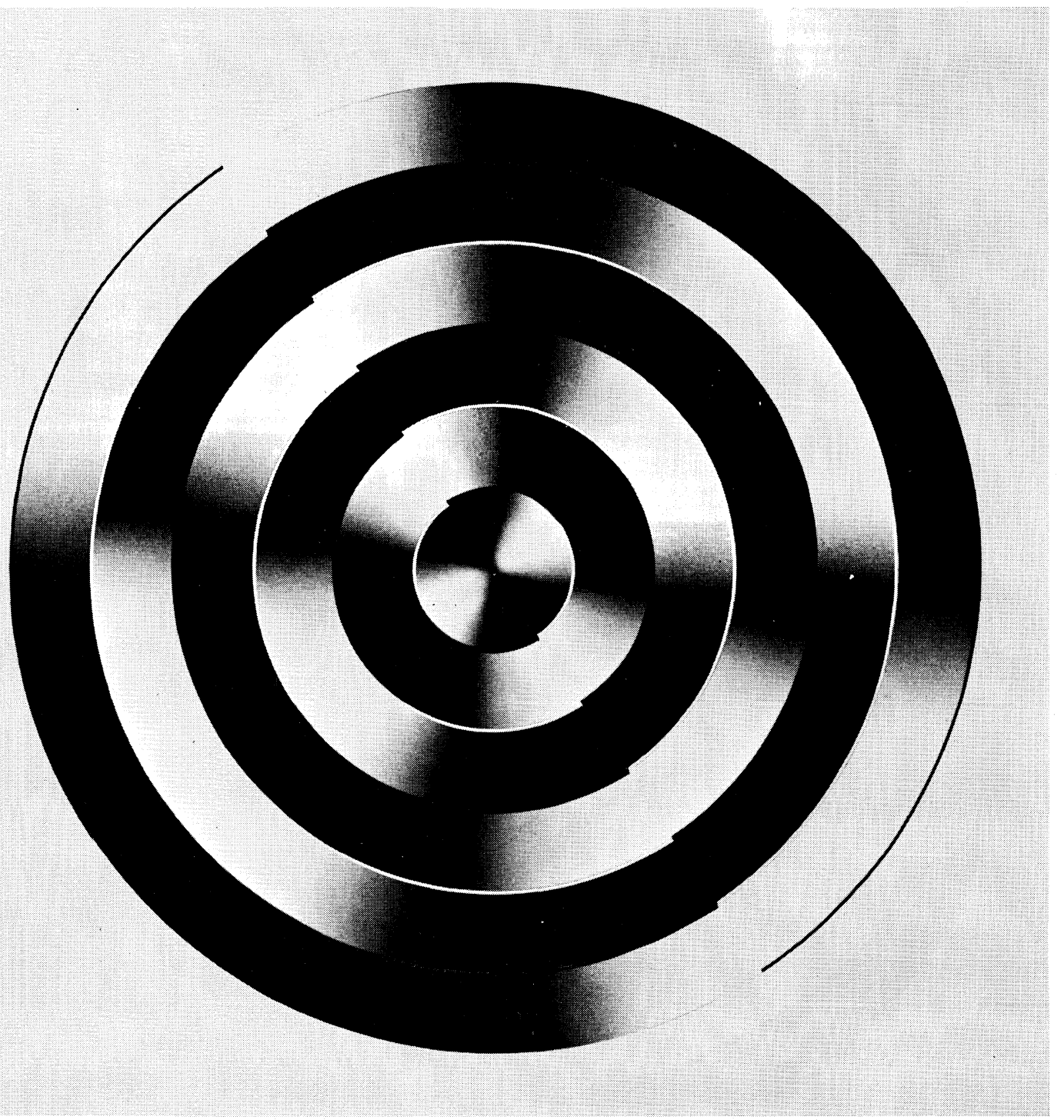

Anstis and Rogers, figure A1: demonstration disc. 


\section{APPENDIX}

Two demonstrations of the four-stroke cycle have been prepared. One is the rotatable disc shown in figure $\mathrm{A} 1$, which the reader is invited to cut out and spin on a record player turntable. The other is a movie, which we shall describe verbally in this appendix.

The disc was designed to give the same visual stimulus as a positive-plus-negative square-wave grating undergoing a four-stroke cycle on a CRO screen. Such a grating consists of alternating light and dark bars, which fade gradually from light to dark or vice versa during the dissolves. The bars of the grating are separated spatially by very narrow white or black fringes, a few minutes visual angle wide. The width of these fringes is equal to the physical displacement between the positive and negative gratings. Since the four-stroke cycle repeats endlessly over time, the disc presents only a single cycle which repeats on each revolution of the disc.

The disc consists of six concentric bands, each as wide as a bar of the grating. The bands are separated by very narrow white or black arcs, which are the fringes. On each revolution the bars fade from light to dark and back, and each fringe jumps through its own width - a few minutes visual angle-twice per revolution, changing sharply from white to black or vice versa as it does so.

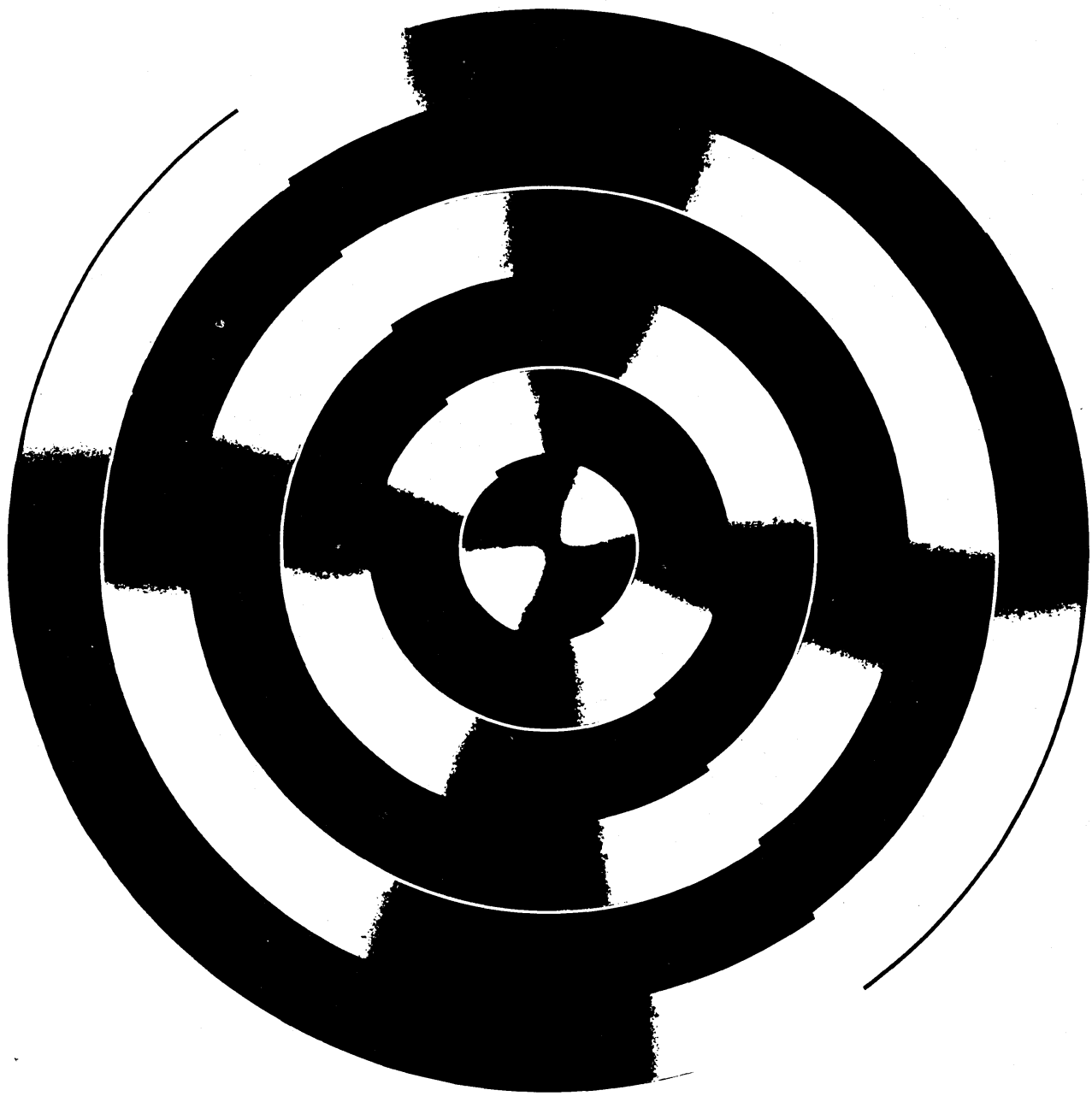


If viewed behind a narrow radial slit, the rotating disc gives approximately the same stimulus as a four-stroke cycling grating on a CRO. In practice, the slit is not necessary, since what is true for any radial slit-shaped region across the disc is true for all other parts of the disc. So when it rotates smoothly, say at $33 \mathrm{rev} \mathrm{min}^{-1}$, the whole disc will appear to be steadily expanding if rotated clockwise, or contracting if rotated anticlockwise. Moreover, the effect is paradoxical, because the concentric circles on the disc can clearly be seen not to change their positions, despite the apparent expansion or contraction. Thus there is a conflict between the position and motion information.

The other demonstration of the four-stroke cycle used $35 \mathrm{~mm}$ slides of pictures instead of gratings, and a rotary motion instead of translation. Three $35 \mathrm{~mm}$ slides were prepared of a black and white engraving by Breughel. Two slides were identical positives and the third was a photographic negative. All three slides were projected from separate projectors on to the same screen, overlapping and almost, but not quite, in register. The negative picture was set to an intermediate luminance and thereafter left alone. To make the positive picture jump back and forth, the two positives were first positioned in exact register with the negative, then one was tilted slightly clockwise and the other an equal amount anticlockwise from the in-register position. Thereafter the positives were never moved, but a moveable shutter was arranged to expose each positive in alternation, so that a single positive was seen jumping back and forth between two rotated positions on the screen. It looked like a single picture rocking back and forth. In addition, each positive picture could be independently dimmed by solid-state dimmers.

By manual operation of the shutter and dimmers in a cycle, the positive picture was made to jump to the right, grow dimmer, jump to the left, and grow brighter. This cycle was superimposed on the static unchanging negative. For convenience the whole display was filmed with a cine camera aimed at the screen and projected later. The result was a compelling illusion that the picture on the screen was continuously rotating clockwise for as long as the cycle was running. Yet fixation upon any contour in the picture revealed a perceptual paradox: although the picture as a whole appeared to rotate, each individual contour could be seen to remain in the same place. The picture appeared to move without getting anywhere.

After about $30 \mathrm{~s}$ viewing of the apparent rotation, the stationary picture was kept on the screen. A strong anticlockwise motion aftereffect could be seen. This indicates that the illusory motion was an adequate stimulus for hard-wired motion detectors in the visual system, and was adapting them enough to produce an aftereffect (Anstis and Cavanagh 1981).

Thus the continuous rotation perceived in the four-stroke movie is very different from the tiny shifts in the stimulus. Also, the percept is itself paradoxical, in that it appears to do two incompatible things at once-it moves steadily in one direction and yet at the same time it stays more or less in the same place. This visual ambiguity suggests that information about motion and about visual direction or position may be coded in separate and relatively independent channels in the human visual system (Anstis 1980). 